\title{
Shell-Isolated Au Nanoparticles Functionalized with Rhodamine B Fluorophores in Helium Nanodroplets
}

\author{
Roman Messner, Wolfgang E. Ernst, and Florian Lackner* \\ Cite This: J. Phys. Chem. Lett. 2021, 12, 145-150 \\ Read Online
}

ABSTRACT: Nanoparticles consisting of three different materials in a layered core@shell@shell structure are synthesized in cold helium droplets by sequential doping. Upon the formation of Au core particles, a first shell layer is formed by adding either Ar, isopropyl alcohol, or hexane. Subsequently, the droplets are doped with rhodamine B (RB) molecules; fluorescence spectra recorded upon laser excitation at $532 \mathrm{~nm}$ provide insight into the structure of the formed complexes. For the two-component $\mathrm{Au} @ \mathrm{RB}$ system, the RB fluorescence is quenched in the presence of the $\mathrm{Au}$ core. If an intermediate isolating shell layer is introduced (Au@shell@RB), the fluorescence increases again. The results demonstrate that shell-isolated nanoparticles can be formed inside He nanodroplets and functionalized in situ with additional molecules. As the structure of the particles depends on the pickup sequence, the approach can be exploited for the synthesis and investigation of a large variety of different combinations of plasmonic metals, intermediate layers, and molecules.

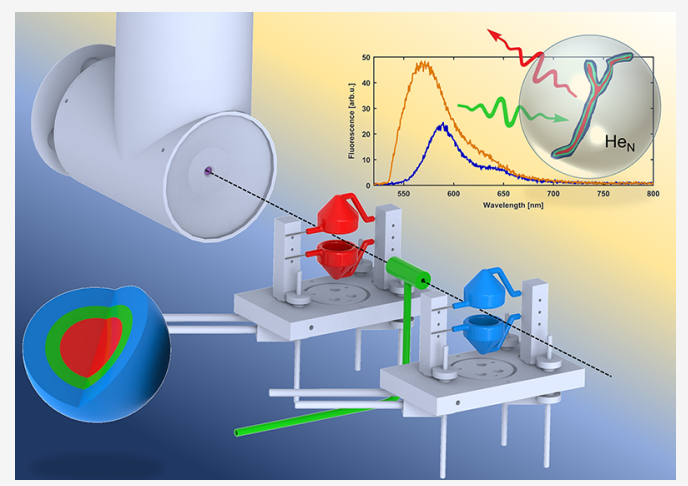

$\mathrm{T}$ he merging of plasmonics and spectroscopy resulted in a fruitful symbiosis from which many applications have emerged, ranging from plasmon-enhanced fluorescence (PEF) and surface plasmon resonance (SPR) spectroscopy to tip- and surface-enhanced Raman spectroscopy (TERS and SERS, respectively). ${ }^{1-7}$ About a decade ago, the versatility of methods based on plasmonic materials was greatly expanded by the introduction of shell-isolated nanoparticles (SHINs). ${ }^{8}$ These particles consist of a plasmonic metal covered by a chemically inert shell layer, which avoids particle agglomeration and direct contact between probe molecules and metal. $^{9-11}$ Considering the widespread applications, an understanding of the interactions between different plasmonic metal nanoparticles and molecules is very important. Here, we introduce helium droplet synthesis as a new bottom-up approach for the formation and investigation of such systems, consisting of plasmonic nanoparticles covered by a shell layer and surrounded by fluorophore molecules.

The optical properties of $\mathrm{Cu}^{12}$ and $\mathrm{Ag}^{13}$ nanoparticles as well as $\mathrm{Au}$ oligomers ${ }^{14}$ inside helium droplets have been studied previously. For spherical Ag particles in small helium droplets, the localized surface plasmon resonance (LSPR) at about $3.8 \mathrm{eV}$ has been observed. With increasing droplet size, the presence of quantum vortices, ${ }^{15,16}$ along which dopants preferentially agglomerate, leads to the formation of metal nanowires. ${ }^{17-19}$ The transition from compact isolated nanoparticles to elongated wire structures goes along with the emergence of additional plasmon modes in the visible and infrared, characteristic for nonspherical structures. ${ }^{20}$ The LSPR of deposited nanoparticles formed by helium droplet synthesis has been investigated for the Ag@Au system. ${ }^{21,22}$

Infrared spectroscopy of $\mathrm{Ag}$ particles covered with shells of ethane, ${ }^{23}$ methane, ethylene, and acetylene ${ }^{24}$ revealed the formation of solid-like (or glassy for ethane) layers around the $\mathrm{Ag}$ core in the cold He droplet environment. Here, we show that such a layer can be used as a spacer between fluorophore molecules and a metal particle, a configuration similar to shellisolated nanoparticle structures. ${ }^{3,25-28}$ Thereby, the cold (0.4 $\mathrm{K}){ }^{29}$ superfluid $\mathrm{He}$ environment allows for the use of unconventional isolation layers (argon, isopropyl alcohol, and hexane), which are combined with rhodamine B fluorophores and Au core particles. The results demonstrate that triple-layer systems can be formed in helium droplets and open up new opportunities for the combination of different materials in the inert helium droplet synthesis environment.

Core@shell@shell particles are formed by the successive capture and coagulation of dopants in three different pickup regions, ${ }^{30}$ as sketched in Figure 1 . The He droplets are first doped with $\mathrm{Au}$ atoms, which agglomerate and form spherical or elongated wire-like metal particles, depending on the initial He droplet size and the pickup oven temperature. ${ }^{31}$

Received: November 14, 2020

Accepted: December 7, 2020

Published: December 14, 2020 


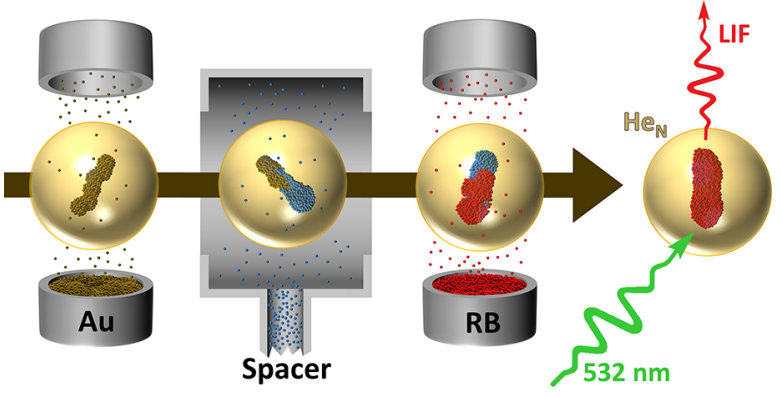

Figure 1. Sketch of the helium droplet synthesis process. Triplelayered particles are formed by sequential doping and analyzed by laser-induced fluorescence (LIF) spectroscopy.

Subsequently, either Ar, isopropyl alcohol (iPrOH), or hexane is added. The last pickup cell passed by the droplet beam holds rhodamine $\mathrm{B}(\mathrm{RB})$ molecules.

We start the discussion with bare $\mathrm{RB}$ molecules isolated in helium droplets. Figure 2a shows LIF spectra recorded for RB

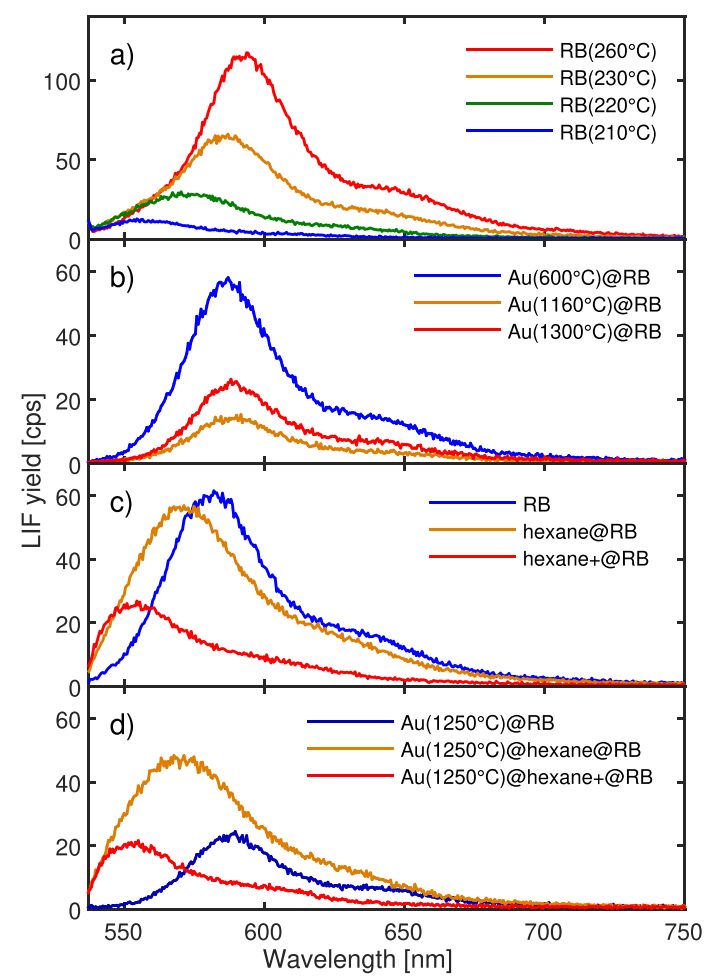

Figure 2. Dispersed LIF spectra recorded using large helium droplets ( $\sim 800 \mathrm{~nm}$ diameter): (a) bare RB, fluorescence as a function of the $\mathrm{RB}$ oven temperature, (b) spectra recorded for different $\mathrm{Au}$ oven temperatures at a constant RB doping level, (c) hexane@RB core@ shell particles for different hexane doping pressures (no Au doping), (d)Au@hexane@RB particles for different hexane doping levels and constant $\mathrm{Au}$ and $\mathrm{RB}$ oven temperatures. The red "hexane+" spectrum has been recorded at a higher hexane partial pressure than the yellow "hexane" spectrum.

in large helium droplets $(d \approx 800 \mathrm{~nm})$ as a function of doping level, controlled by the temperature of the RB pickup oven. The molecules are excited with a $532 \mathrm{~nm}$ continuous-wave laser, resonant to the $\pi-\pi^{*}$ transition. With increasing oven temperature, a red-shift of the peak is observed. While at high temperatures the peak form is very similar to $\mathrm{RB}$ thin films ${ }^{32}$ or $\mathrm{RB}$ in solution, ${ }^{33}$ at a low doping level, the spectrum is more similar to the spectra of gas-phase molecules. ${ }^{34,35}$ (see Supporting Information (SI), Figure S6 for a comparison to literature spectra). The observed variation of the peak position from 550 to $593 \mathrm{~nm}$ is explained as follows: At low oven temperatures, the number of molecules per droplet is small, and large complexes cannot be formed. In particular, if multiple vortices are present, as expected at the employed droplet sizes, ${ }^{16}$ molecules may be attached to them at different sites, ${ }^{36}$ giving rise to a free-molecule-like fluorescence due to the only weak perturbation by the droplet environment. With increasing doping level, RB molecules start to coagulate, which goes along with a more thin-film- or solution-like fluorescence. Thus, the observed change in the spectra with increasing doping level is interpreted as an evolution from isolated molecules or small complexes pinned to vortices to a regime where $\mathrm{RB}$ molecules are agglomerated and form large elongated wire structures. ${ }^{31}$

The results presented in the following are obtained at a constant $\mathrm{RB}$ oven temperature of about $230{ }^{\circ} \mathrm{C}$, a temperature at which degradation effects due to the thermal decomposition of molecules do not affect the LIF signal (see Experimental Methods for details). ${ }^{37}$ At this temperature, the fluorescence peak is located at about $586 \mathrm{~nm}$.

Figure $2 \mathrm{~b}$ shows three dispersed LIF spectra of $\mathrm{Au} @ \mathrm{RB}$ core@shell particles recorded for different Au oven temperatures, while the RB temperature is kept constant. Note that at $600{ }^{\circ} \mathrm{C}$, Au doping is negligible, and the spectrum provides a reference that corresponds to bare $\mathrm{RB}$ complexes. It becomes evident that the shape and position of the fluorescence spectrum is almost unaffected by the presence of the $\mathrm{Au}$ particles; only a weak blue-shift of about $2 \mathrm{~nm}$ is observed. However, the signal intensity decreases significantly with increasing Au amount. The results have been obtained for large droplets $(d \approx 800 \mathrm{~nm})$ that host vortices. For $\mathrm{Au}$, it has been shown that in this case, small isolated $\mathrm{Au}$ particles are formed at low doping levels, whereas elongated nanowires, pinned to vortices, are present at high doping levels. ${ }^{31}$ For the recorded spectra, this implies that at low doping levels, small spherical $\mathrm{Au}$ clusters are present prior to $\mathrm{RB}$ doping, while at high oven temperatures, $\mathrm{Au}$ nanowires are present, to which $\mathrm{RB}$ molecules can attach. Furthermore, in the synthesis of multilayered structures in helium droplets, one has to keep in mind that for every atom that is added, the droplet size is reduced by the evaporation of $\mathrm{He}$ atoms in order to dissipate the excess binding energy. By this mechanism, the droplets can keep their temperature at $0.4 \mathrm{~K}$ constant; however, it also causes a reduction of the geometric cross section and limits, thus, the amount of shell material that can be added to a core particle. Consequently, in the absence of any additional effects, one would expect a continuous decrease of the fluorescence yield with an increasing amount of $\mathrm{Au}$ core material for a constant $\mathrm{RB}$ oven temperature.

To investigate the effect of the Au core on the fluorescence in more detail, the total RB fluorescence yield as a function of the Au oven temperature is shown in Figure 3. Each data point has been obtained by integrating the recorded LIF spectrum from 530 to $750 \mathrm{~nm}$. We start the discussion with Au@RB core@shell particles in large helium droplets $(6 \mathrm{~K}$ nozzle temperature, $d \approx 800 \mathrm{~nm}$ ). As soon as $\mathrm{Au}$ is present, the fluorescence is quenched, reflected by the rapid decrease of the fluorescence with increasing $\mathrm{Au}$ temperature. Fluorescence quenching is very typical for fluorophores directly attached to a metal surface. ${ }^{3,25-28}$ Au nanoparticles are known to be efficient 


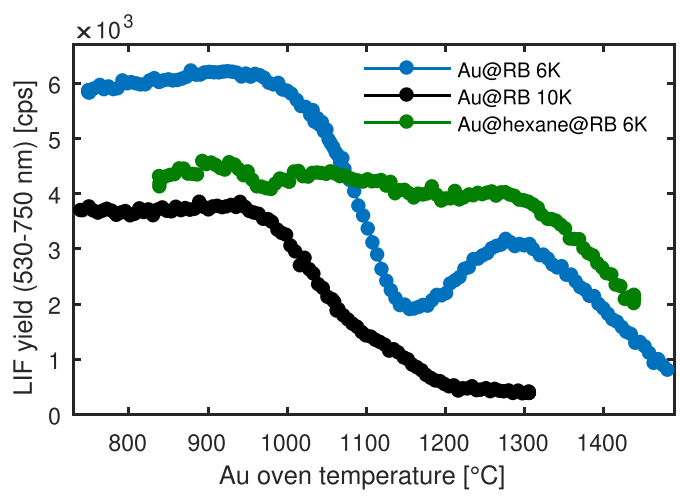

Figure 3. Integrated fluorescence yield of core@shell and core@ shell@shell nanoparticles as a function of the Au oven temperature. Blue: Au@RB particles in large $(6 \mathrm{~K}, d \approx 800 \mathrm{~nm})$ He droplets; black: $\mathrm{Au} @ \mathrm{RB}$ particles in small $(10 \mathrm{~K}, d \approx 80 \mathrm{~nm}) \mathrm{He}$ droplets; green: $\mathrm{Au} @$ hexane@RB in large $(6 \mathrm{~K}, d \approx 800 \mathrm{~nm})$ He droplets.

quenchers for fluorescent dyes; in particular, fluorescence quenching has been observed for RB molecules adsorbed on citrate capped $\mathrm{Au}$ nanoparticles. ${ }^{38,39}$ This observation indicates that the molecules are in contact with the metal core inside the helium droplet, i.e., core@shell Au@RB structures are formed. The fluorescence decreases continuously up to about $1150{ }^{\circ} \mathrm{C}$, a temperature where the helium droplets are still only sparsely doped. Interestingly, the fluorescence recovers and shows a local maximum at $1280{ }^{\circ} \mathrm{C}$. At this point, the He droplets have only lost about $5 \%$ of their volume, as estimated from the $\mathrm{He}$ background pressure. ${ }^{31}$ The final decrease of the fluorescence yield beyond $1280{ }^{\circ} \mathrm{C}$ is attributed to the continuous shrinking of the $\mathrm{He}$ droplets with increasing $\mathrm{Au}$ doping. This correlation between droplet beam attenuation and fluorescence decrease is discussed in more detail in the SI (Figure S7). The black curve shows the same experiment but for small He droplets $(10 \mathrm{~K}$ nozzle temperature, $d \approx 80 \mathrm{~nm})$, in which spherical $(<5 \mathrm{~nm})$ $\mathrm{Au}$ particles constitute the core material. The trend is similar in this case: a rapid monotonic decrease is observed with only a weak shoulder at about $1100{ }^{\circ} \mathrm{C}$.

In the case of large $\mathrm{He}$ droplets, the fluorescence enhancement with respect to the local minimum is attributed to the structural change from individual small Au clusters to connected nanowires with increasing pickup level. Small separated Au clusters have a larger surface area than elongated wire structures despite the fact that there are more metal atoms present in the second case. If the $\mathrm{Au}$ surface decreases, fewer $\mathrm{RB}$ molecules can attach to the $\mathrm{Au}$, and thus, the number of particles affected by surface quenching is lower. For small droplets, this effect is also present but not as pronounced, because long elongated wires are not formed. A significant plasmon-enhancement effect is not expected due to the close proximity between molecules and metal. ${ }^{27}$ However, for $\mathrm{Au}$ nanoparticles, it has been shown that only particles larger than about $2 \mathrm{~nm}$ support an LSPR due to quantum confinement effects. ${ }^{40}$ Thus, the emerging LSPR may contribute to the signal with increasing Au particle size.

A common approach to reduce the fluorescence quenching in metal-molecule systems is based on the insertion of a nonconducting dielectric shell layer between the metal and fluorophore. The resulting structures are referred to as shellisolated nanoparticles (SHINs). ${ }^{8}$ This requires the formation of triple-layer nanoparticles, which has so far not been realized with the helium droplet approach. In order to demonstrate this concept, we use a third pickup cell mounted between the $\mathrm{Au}$ and RB pickup regions. In our current experimental setup, geometrical restrictions allow only for the implementation of a cell that holds gaseous species; however, in principle, a large variety of dopants can be used as a shell layer if a third high temperature oven is installed. Thus, $\mathrm{Ar}, \mathrm{iPrOH}$, and hexane have been employed as spacer material, all of which are expected to form a solid shell layer around the $\mathrm{Au}$ structures inside the droplets at $0.4 \mathrm{~K}^{23,24,41}$ If the configuration results indeed in Au@hexane@RB,Au@iPrOH@RB, and Au@Ar@ RB core@shell@shell nanoparticles, the intermediate layer will separate the molecules from the metal, thereby, reducing the effect of fluorescence quenching.

It is instructive to start the discussion with the spectra of hexane@RB nanoparticles without the Au core, which are presented in Figure 2c, recorded without hexane (blue), with low hexane (orange), and heavy hexane (red) doping. It can be seen that the addition of a hexane "core" is accompanied by an increasing blue-shift and a decrease of the fluorescence yield. The latter is explained by the shrinking of the He droplet upon doping with hexane.

Figure 2d shows spectra for hexane concentrations comparable to Figure $2 \mathrm{c}$ but with an additional $\mathrm{Au}$ core. Comparing the spectra with (orange) and without (blue) hexane shows that the presence of the isolation layer enhances the total fluorescence yield. This observation indicates that inside a helium droplet, the addition of hexane leads to the formation of an intermediate spacer shell that inhibits direct contact between RB and Au metal. However, the enhancement goes along with a prominent blue-shift. At heavy hexane doping levels, the shrinking of the $\mathrm{He}$ droplets due to the hexane doping dominates, and the spectrum is very similar to the one without a Au core (red spectrum, Figure 2c).

The green trace in Figure 3 shows the effect of moderate hexane doping on the integrated fluorescence yield of $\mathrm{Au} @$ hexane@RB structures in large He droplets as a function of $\mathrm{Au}$ oven temperature. In contrast to the $A u @ R B$ traces, an immediate quenching is not observed upon Au doping, indicating that the $\mathrm{RB}$ molecules do not get in contact with the metal surface if hexane is present. The signal remains fairly constant and starts to decrease beyond $1300{ }^{\circ} \mathrm{C}$ where the effect of the decreasing droplet size, i.e., pickup cross section, takes over. An increase of the fluorescence with respect to the base level, however, is not observed, similar to previous experiments on $\mathrm{RB}$ molecules attached to $\mathrm{Au}$ particles in solution. ${ }^{38,39}$ In the case of small molecule-particle separation, the overlap of the Au LSPR with the RB emission enables an effective energy transfer from the molecule to the Au particle, reducing the $\mathrm{RB}$ fluorescence. For very small particles, the energy transfer proceeds through nanosurface energy transfer (NSET) (non-Förster energy transfer). ${ }^{38,42}$

Figure 4 shows a series of experiments with Au@spacer@RB particles using three different spacer materials: $\mathrm{iPrOH}, \mathrm{Ar}$, and hexane. The amount of spacer molecules is continuously increased by slowly opening a leak valve connected to a reservoir that holds the molecules in the gas phase. In this series of experiments, the doping conditions for $\mathrm{Au}$ and $\mathrm{RB}$ are kept constant. At the position marked by the dashed line, the valve is closed again, and the pressure in the intermediate pickup cell slowly decreases again. Each red data point corresponds to the integrated fluorescence yield (530 to 750 $\mathrm{nm})$. It can be seen that all three spacer materials show the same behavior, i.e., the fluorescence yield is first enhanced and 


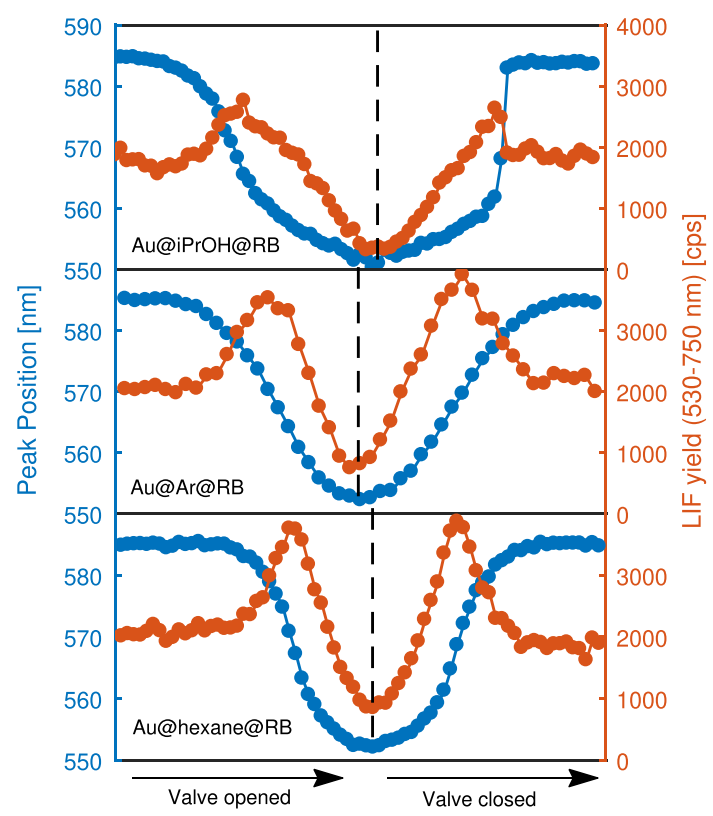

Figure 4. Integrated fluorescence yields (red) and peak positions (blue) for Au@iPrOH@RB, Au@Ar@RB, and Au@hexane@RB nanoparticles (Au oven at $1310{ }^{\circ} \mathrm{C}$ ). The leak valve is slowly opened until the dashed line and then closed again.

decreases again with increasing doping level. The effect is most prominent for $\mathrm{Ar}$ and hexane, which both show a 2 -fold enhancement compared to the baseline, whereas for $\mathrm{iPrOH}$, the yield increases by a factor of 1.5 . This may be explained by differences in the interaction between spacer molecules and RB. In additional measurements, presented in the SI (Figure S2), it has been confirmed that this behavior is only observed for core@shell@shell particles where the metallic core is present, i.e., hexane@RB particles do not show any enhancement. For small triple-layered spherical nanoparticles, the same effect as in Figure 4 is observed (see also SI, Figure S3).

The blue data points correspond to the fluorescence peak position (emission maximum). Without a spacer, the peak position is located at about $586 \mathrm{~nm}$. Interestingly, the peak shifts monotonically to the blue with an increasing doping level, independent of the spacer material. At the fluorescence yield maximum, the peak is blue-shifted by about $15 \mathrm{~nm}$ in all three cases; the shift is continuous and reaches a minimum at about $550 \mathrm{~nm}$. As the behavior is similar for all three molecules, a typical solution shift, which would be different for each spacer material, appears unlikely. ${ }^{33,43,44}$ In the presence of an isolating shell layer, the surface to which RB molecules can attach is larger, and thus, they are more separated from each other. Furthermore, the orientation of the molecules with respect to each other and the metal surface can change. Thus, structural effects may explain the variation of the peak position. However, the peak shift is evidently related to the spacer material, because the same behavior is observed in the absence of the $\mathrm{Au}$ core (Figure 2c).

In this manuscript, we show that triple-layer core@shell@ shell nanoparticles can be formed inside $\mathrm{He}$ droplets by the sequential addition of different dopants. Rhodamine B molecules are employed as fluorophores, and the recorded laser-induced fluorescence (LIF) spectra provide insight into the configuration of the core and shell layers inside the droplets. In the presence of $\mathrm{Au}$ core particles, fluorescence quenching is observed, similar to dye molecules attached to $\mathrm{Au}$ nanoparticles in solution. ${ }^{38,39}$ If an intermediate shell layer is inserted between the $\mathrm{Au}$ core and $\mathrm{RB}$ molecules, the fluorescence increases again. This behavior is observed for three different spacer molecules, hexane, $\mathrm{iPrOH}$, and $\mathrm{Ar}$, all of which are expected to be solid at the $0.4 \mathrm{~K} \mathrm{He}$ droplet temperature. This indicates that the additional intermediate layer acts as an isolating shell that separates the metal from the $\mathrm{RB}$ molecules. It is, thus, concluded that core@shell@shell nanoparticles are formed inside He dropelts, similar to shellisolated nanoparticles (SHINs) that are used in surfaceenhanced spectroscopy methods. ${ }^{8}$

In the case of Raman spectroscopy, fluorescence quenching is advantageous, as it eases the detection of Raman scattered light. Thus, by exploiting plasmonic enhancement, it may become possible to employ Raman spectroscopy as a new analysis tool to probe molecules and nanoparticles in helium droplets.

Considering that helium droplets can be doped with a large variety of materials, systematic studies of different molecule, spacer, and plasmonic metal combinations may lead to new materials with potential applications in sensor technologies $^{5,6,45}$ or biomedicine. ${ }^{46}$

\section{EXPERIMENTAL METHODS}

The nanoparticles are synthesized in helium droplets by sequential pickup of dopant atoms and molecules; the employed apparatus is described in detail in ref 47. Rhodamine B from Radiant Dyes Laser $\mathrm{GmbH}$, Au pellets (99.9\%) from Ögussa GmbH, Ar gas (99.999\%) from Tega GmbH, and isopropyl alcohol (99.7\%) from Merck $\mathrm{GmbH}$ are used as doping material without further treatment.

The expansion of $\mathrm{He}$ (99.9999\%, from Air Liquide Austria $\mathrm{GmbH}$ ) at a pressure of 60 bar through a $5 \mu \mathrm{m}$ nozzle at 6 and $10 \mathrm{~K}$ results in $\mathrm{He}$ droplets with an estimated diameter of about 800 and $80 \mathrm{~nm}$, respectively, corresponding to $\sim 1 \cdot 10^{10}$ and $\sim 7 \cdot 10^{6} \mathrm{He}$ atoms per droplet. ${ }^{29,48}$ The droplet beam passes two pickup ovens, the first loaded with Au metal and the second holding rhodamine $B(R B)$ powder. The $R B$ oven is kept below $245^{\circ} \mathrm{C}$ to prevent thermal decomposition, ${ }^{37}$ except for the temperature study presented in Figure $2 b$, during which $260{ }^{\circ} \mathrm{C}$ has been reached. If the $\mathrm{RB}$ temperature is kept at such a high temperature for time scales on the order of hours, a degradation of the molecules is observed in the form of a continuous decrease of the LIF signal intensity. Furthermore, the $\mathrm{RB}$ powder in the pickup oven was replaced after each series of experiments. The RB pickup has been investigated in more detail; time-of-flight (TOF) mass spectra are presented and discussed in the SI (Figure S1). A strong RB parent peak ${ }^{49}$ is observed, indicating intact molecules after evaporation. The $\mathrm{RB}$ oven temperatures stated in the manuscript are estimated based on the heating current and the oven resistance. For $\mathrm{Au}$, the melting plateau is visible in LIF spectra recorded as a function of the $\mathrm{Au}$ oven temperature, providing a reference point for the Au temperature scale. The corresponding spectra in the main manuscript are calibrated and have been obtained by the procedure described in the SI (see Figure S5).

A gas pickup cell is mounted in between these two ovens, allowing for doping with a third species that can be introduced via a precision leak valve. This setup requires that the intermediate dopant is either gaseous or that it can be easily evaporated, which is the case for the used species hexane, Ar, and $\mathrm{iPrOH}$. 
Two different droplet sizes (nozzle temperatures) have been chosen in order to access different nanoparticle sizes and shapes. At a nozzle temperature of $6 \mathrm{~K}$, large elongated nanoparticle structures can be formed inside helium droplets, consisting of more than $10^{5}$ dopants. At $10 \mathrm{~K}$, spherical structures are formed, consisting of several hundreds to thousands of dopants with diameters up to $5 \mathrm{~nm} .^{31}$

The prepared particles are analyzed in situ under UHV conditions by dispersed laser-induced fluorescence (LIF) spectroscopy in a separately pumped chamber using a Kymera $328 \mathrm{i}$ spectrograph equipped with a cooled iDus $401 \mathrm{CCD}$ camera $\left(-100{ }^{\circ} \mathrm{C}\right)$. An edge filter (Semrock 532 RazorEdge) is used for laser stray-light suppression. The entry slit opening of the spectrograph has been set to $150 \mu \mathrm{m}$, and a 150 lines $/ \mathrm{mm}$ $(500 \mathrm{~nm})$ diffraction grating was used. All spectra were captured with a $20 \mathrm{~s}$ acquisition time. A Coherent Verdi V18 provides $532 \mathrm{~nm}$ continuous-wave laser light to excite the RB molecules; $5 \mathrm{~W}$ (focus diameter $\approx 0.5 \mathrm{~mm}$ ) has been employed in the experiments (see SI, Figure S4, for laser power and saturation studies).

\section{ASSOCIATED CONTENT}

\section{SI Supporting Information}

The Supporting Information is available free of charge at https://pubs.acs.org/doi/10.1021/acs.jpclett.0c03399.

Time-of-flight mass spectra of RB doped He droplets, additional integrated fluorescence yield traces for hexane@RB and small Au@hexane@RB particles, laser power dependence studies of the fluorescence yield, an explanation of the $\mathrm{Au}$ oven temperature calibration as well as a comparison of RB LIF spectra with literature spectra (PDF)

\section{AUTHOR INFORMATION}

\section{Corresponding Author}

Florian Lackner - Institute of Experimental Physics, Graz University of Technology, A-8010 Graz, Austria/EU; ○ orcid.org/0000-0003-3047-5659;

Email: florian.lackner@tugraz.at

\section{Authors}

Roman Messner - Institute of Experimental Physics, Graz University of Technology, A-8010 Graz, Austria/EU

Wolfgang E. Ernst - Institute of Experimental Physics, Graz University of Technology, A-8010 Graz, Austria/EU; (1) orcid.org/0000-0001-8849-5658

Complete contact information is available at:

https://pubs.acs.org/10.1021/acs.jpclett.0c03399

\section{Notes}

The authors declare no competing financial interest.

\section{ACKNOWLEDGMENTS}

The authors acknowledge support by NAWI Graz and the Austrian Science Fund (FWF) grant P30940-N39.

\section{REFERENCES}

(1) Dong, J.; Zhang, Z.; Zheng, H.; Sun, M. Recent Progress on Plasmon-Enhanced Fluorescence. Nanophotonics 2015, 4, 472-490.

(2) Dong, J.; Gao, W.; Han, Q.; Wang, Y.; Qi, J.; Yan, X.; Sun, M. Plasmon-Enhanced Upconversion Photoluminescence: Mechanism and Application. Rev. Phys. 2019, 4, 100026.
(3) Li, J.-F.; Li, C.-Y.; Aroca, R. F. Plasmon-Enhanced Fluorescence Spectroscopy. Chem. Soc. Rev. 2017, 46, 3962-3979.

(4) Zong, H.; Wang, X.; Mu, X.; Wang, J.; Sun, M. PlasmonEnhanced Fluorescence Resonance Energy Transfer. Chem. Rec. 2019, 19, 818-842.

(5) Bauch, M.; Toma, K.; Toma, M.; Zhang, Q.; Dostalek, J. Plasmon-Enhanced Fluorescence Biosensors: A Review. Plasmonics 2014, 9, 781-799.

(6) Li, M.; Cushing, S. K.; Wu, N. Plasmon-Enhanced Optical Sensors: A Review. Analyst 2015, 140, 386-406.

(7) Willets, K. A.; Van Duyne, R. P. Localized Surface Plasmon Resonance Spectroscopy and Sensing. Annu. Rev. Phys. Chem. 2007, 58, 267-297.

(8) Li, J. F.; Huang, Y. F.; Ding, Y.; Yang, Z. L.; Li, S. B.; Zhou, X. S.; Fan, F. R.; Zhang, W.; Zhou, Z. Y.; Wu, D. Y.; et al. Shell-Isolated Nanoparticle-Enhanced Raman Spectroscopy. Nature 2010, 464, 392-395.

(9) Asselin, J.; Legros, P.; Grégoire, A.; Boudreau, D. Correlating Metal-Enhanced Fluorescence and Structural Properties in $\mathrm{Ag} @ \mathrm{SiO}_{2}$ Core-Shell Nanoparticles. Plasmonics 2016, 11, 1369-1376.

(10) Aroca, R. F.; Teo, G. Y.; Mohan, H.; Guerrero, A. R.; Albella, P.; Moreno, F. Plasmon-Enhanced Fluorescence and Spectral Modification in SHINEF. J. Phys. Chem. C 2011, 115, 20419-20424.

(11) Guerrero, A. R.; Aroca, R. F. Surface-Enhanced Fluorescence with Shell-Isolated Nanoparticles (SHINEF). Angew. Chem., Int. Ed. 2011, 50, 665-668.

(12) Gomez, L. F.; Loginov, E.; Halder, A.; Kresin, V. V.; Vilesov, A. F. Formation of Unusual Copper Clusters in Helium Nanodroplets. Int. J. Nanosci. 2013, 12, 1350014.

(13) Loginov, E.; Gomez, L. F.; Chiang, N.; Halder, A.; Guggemos, N.; Kresin, V. V.; Vilesov, A. F. Photoabsorption of $\operatorname{Ag}_{\mathrm{N}}\left(\mathrm{N}_{\sim} 6-6000\right)$ Nanoclusters Formed in Helium Droplets: Transition from Compact to Multicenter Aggregation. Phys. Rev. Lett. 2011, 106, 233401.

(14) Messner, R.; Schiffmann, A.; Pototschnig, J. V.; Lasserus, M.; Schnedlitz, M.; Lackner, F.; Ernst, W. E. Spectroscopy of Gold Atoms and Gold Oligomers in Helium Nanodroplets. J. Chem. Phys. 2018, 149, 024305.

(15) Gessner, O.; Vilesov, A. F. Imaging Quantum Vortices in Superfluid Helium Droplets. Annu. Rev. Phys. Chem. 2019, 70, 173198.

(16) Gomez, L. F.; Ferguson, K. R.; Cryan, J. P.; Bacellar, C.; Tanyag, R. M. P.; Jones, C.; Schorb, S.; Anielski, D.; Belkacem, A.; Bernando, C.; et al. Shapes and Vorticities of Superfluid Helium Nanodroplets. Science 2014, 345, 906-909.

(17) Latimer, E.; Spence, D.; Feng, C.; Boatwright, A.; Ellis, A. M.; Yang, S. Preparation of Ultrathin Nanowires Using Superfluid Helium Droplets. Nano Lett. 2014, 14, 2902-2906.

(18) Gomez, L. F.; Loginov, E.; Vilesov, A. F. Traces of Vortices in Superfluid Helium Droplets. Phys. Rev. Lett. 2012, 108, 155302.

(19) Thaler, P.; Volk, A.; Lackner, F.; Steurer, J.; Knez, D.; Grogger, W.; Hofer, F.; Ernst, W. E. Formation of Bimetallic Core-Shell Nanowires along Vortices in Superfluid He Nanodroplets. Phys. Rev. B: Condens. Matter Mater. Phys. 2014, 90, 155442.

(20) Gomez, L. F.; O'Connell, S. M. O.; Jones, C. F.; Kwok, J.; Vilesov, A. F. Laser-Induced Reconstruction of Ag Clusters in Helium Droplets. J. Chem. Phys. 2016, 145, 114304.

(21) Lackner, F.; Schiffmann, A.; Lasserus, M.; Messner, R.; Schnedlitz, M.; Fitzek, H.; Pölt, P.; Knez, D.; Kothleitner, G.; Ernst, W. E. Helium Nanodroplet Assisted Synthesis of Bimetallic Ag@Au Nanoparticles with Tunable Localized Surface Plasmon Resonance. Eur. Phys. J. D 2019, 73, 104.

(22) Schiffmann, A.; Knez, D.; Lackner, F.; Lasserus, M.; Messner, R.; Schnedlitz, M.; Kothleitner, G.; Hofer, F.; Ernst, W. E. Ultra-Thin h-BN Substrates for Nanoscale Plasmon Spectroscopy. J. Appl. Phys. 2019, 125, 023104.

(23) Loginov, E.; Gomez, L. F.; Vilesov, A. F. Formation of CoreShell Silver-Ethane Clusters in He Droplets. J. Phys. Chem. A 2013, 117, 11774-11782. 
(24) Loginov, E.; Gomez, L. F.; Sartakov, B. G.; Vilesov, A. F. Formation of Large Ag Clusters with Shells of Methane, Ethylene, and Acetylene in He Droplets. J. Phys. Chem. A 2016, 120, 6738-6744.

(25) Fort, E.; Grésillon, S. Surface Enhanced Fluorescence. J. Phys. D: Appl. Phys. 2008, 41, 013001.

(26) Geddes, C. D.; Lakowicz, J. R. Editorial: Metal-Enhanced Fluorescence. J. Fluoresc. 2002, 12, 121-129.

(27) Anger, P.; Bharadwaj, P.; Novotny, L. Enhancement and Quenching of Single-Molecule Fluorescence. Phys. Rev. Lett. 2006, 96, 113002 .

(28) Taylor, A. B.; Zijlstra, P. Single-Molecule Plasmon Sensing: Current Status and Future Prospects. ACS Sens 2017, 2, 1103-1122.

(29) Toennies, J. P.; Vilesov, A. F. Superfluid Helium Droplets: A Uniquely Cold Nanomatrix for Molecules and Molecular Complexes. Angew. Chem., Int. Ed. 2004, 43, 2622-2648.

(30) Ernst, W. E.; Hauser, A. W. Metal Clusters Synthesized in Helium Droplets: Structure and Dynamics from Experiment and Theory. Phys. Chem. Chem. Phys. 2020, Advance article.

(31) Volk, A.; Thaler, P.; Knez, D.; Hauser, A. W.; Steurer, J.; Grogger, W.; Hofer, F.; Ernst, W. E. The Impact of Doping Rates on the Morphologies of Silver and Gold Nanowires Grown in Helium Nanodroplets. Phys. Chem. Chem. Phys. 2016, 18, 1451-1459.

(32) Kemnitz, K.; Tamai, N.; Yamazaki, I.; Nakashima, N.; Yoshihara, K. Fluorescence Decays and Spectral Properties of Rhodamine B in Submono-, Mono-, and Multilayer Systems. J. Phys. Chem. 1986, 90, 5094-5101.

(33) Bojarski, C.; Żurkowska, G.; Tyrzyk, J. The Concentrational Changes of the Fluorescence Decay Time of Rhodamine B in Ethanol. Z. Naturforsch., A: Phys. Sci. 1982, 37, 74-77.

(34) Greisch, J.-F.; Harding, M. E.; Kordel, M.; Klopper, W.; Kappes, M. M.; Schooss, D. Intrinsic Fluorescence Properties of Rhodamine Cations in Gas-Phase: Triplet Lifetimes and Dispersed Fluorescence Spectra. Phys. Chem. Chem. Phys. 2013, 15, 8162-8170.

(35) Sagoo, S. K.; Jockusch, R. A. The Fluorescence Properties of Cationic Rhodamine B in the Gas Phase. J. Photochem. Photobiol., A 2011, 220, 173-178.

(36) Coppens, F.; Ancilotto, F.; Barranco, M.; Halberstadt, N.; Pi, M. Capture of $\mathrm{Xe}$ and $\mathrm{Ar}$ Atoms by Quantized Vortices in ${ }^{4} \mathrm{He}$ Nanodroplets. Phys. Chem. Chem. Phys. 2017, 19, 24805-24818.

(37) Safety Data Sheet for Rhodamine B; Merck KGaA: Darmstadt, Germany, 2017. https://www.merckmillipore.com/AT/en/product/ msds/MDA_CHEM-107599.

(38) Stobiecka, M.; Hepel, M. Multimodal Coupling of Optical Transitions and Plasmonic Oscillations in Rhodamine B Modified Gold Nanoparticles. Phys. Chem. Chem. Phys. 2011, 13, 1131-1139.

(39) Franzen, S.; Folmer, J. C. W.; Glomm, W. R.; O’Neal, R. Optical Properties of Dye Molecules Adsorbed on Single Gold and Silver Nanoparticles. J. Phys. Chem. A 2002, 106, 6533-6540.

(40) Zhou, M.; Zeng, C.; Chen, Y.; Zhao, S.; Sfeir, M. Y.; Zhu, M.; Jin, R. Evolution from the Plasmon to Exciton State in LigandProtected Atomically Precise Gold Nanoparticles. Nat. Commun. 2016, 7, 13240.

(41) Diederich, T.; Tiggesbäumker, J.; Meiwes-Broer, K.-H. Spectroscopy on Rare Gas-Doped Silver Clusters in Helium Droplets. J. Chem. Phys. 2002, 116, 3263-3269.

(42) Li, M.; Cushing, S. K.; Wang, Q.; Shi, X.; Hornak, L. A.; Hong, Z.; Wu, N. Size-Dependent Energy Transfer between CdSe/ZnS Quantum Dots and Gold Nanoparticles. J. Phys. Chem. Lett. 2011, 2, 2125-2129.

(43) Pappalardo, R.; Ahmed, S. Emission from Dyes in the Vapor Phase and the Possibility of Vapor-Phase Dye Lasers. J. Chem. Phys. 1972, 56, 5135-5143.

(44) Fikry, M.; Omar, M. M.; Ismail, L. Z. Effect of Host Medium on the Fluorescence Emission Intensity of Rhodamine B in Liquid and Solid Phase. Modern Trends in Physics Research 2008, 210-219.

(45) Anker, J. N.; Hall, W. P.; Lyandres, O.; Shah, N. C.; Zhao, J.; Van Duyne, R. P. Biosensing with plasmonic nanosensors. Nanoscience and Technology 2009, 308-319.
(46) Webb, J. A.; Bardhan, R. Emerging Advances in Nanomedicine with Engineered Gold Nanostructures. Nanoscale 2014, 6, 25022530.

(47) Thaler, P.; Volk, A.; Knez, D.; Lackner, F.; Haberfehlner, G.; Steurer, J.; Schnedlitz, M.; Ernst, W. E. Synthesis of Nanoparticles in Helium Droplets-A Characterization Comparing Mass-Spectra and Electron Microscopy Data. J. Chem. Phys. 2015, 143, 134201.

(48) Gomez, L. F.; Loginov, E.; Sliter, R.; Vilesov, A. F. Sizes of Large He Droplets. J. Chem. Phys. 2011, 135, 154201.

(49) Ferreira, B. R. V.; Correa, D. N.; Eberlin, M. N.; Vendramini, P. $\mathrm{H}$. Fragmentation Reactions of Rhodamine B and 6G as Revealed by High Accuracy Orbitrap Tandem Mass Spectrometry. J. Braz. Chem. Soc. 2017, 28, 136-142. 